\title{
Comparative Studies on the Interaction Between the Medicine Small Molecule with Pepsin by Fluorescence Quenching Spectroscopy and Improved Spectroscopy
}

\author{
Hongcai Zhang, Baosheng Liu *, Xu Cheng, Jinju Wang \\ College of Chemistry \& Environmental Science, Hebei University, Baoding, P. R. China
}

Email address:

lbs@hbu.edu.cn (Baosheng Liu)

${ }^{*}$ Corresponding author

\section{To cite this article:}

Hongcai Zhang, Baosheng Liu, Xu Cheng, Jinju Wang. Comparative Studies on the Interaction Between the Medicine Small Molecule with Pepsin by Fluorescence Quenching Spectroscopy and Improved Spectroscopy. Journal of Drug Design and Medicinal Chemistry. Vol. 4, No. 1, 2018, pp. 5-9. doi: 10.11648/j.jddmc.20180401.12

Received: May 4, 2018; Accepted: June 7, 2018; Published: June 28, 2018

\begin{abstract}
The binding mechanism between cefetamet pivoxil (CFP) and pepsin (PEP) at different temperatures (298 K, 303 $\mathrm{K}, 310 \mathrm{~K}$ ) was investigated by the classical fluorescence spectroscopy with focus on the fluorescence change of protein, as well as the improved spectroscopy with focus on the fluorescence changes of the resonance light scattering of small molecule drugs. The results showed that the main quenching mode of PEP-CFP was static quenching. The value of $\mathrm{n}$ was approximately equal to 1 which indicating that there was only one binding site in the interaction between PEP and CFP and the Hill coefficient was about 1 which indicating that there was no cooperative between the receptor PEP and ligand CFP. The binding constants of the PEP-CFP system obtained by the improved spectroscopic method were two orders of magnitude larger than that of the traditional fluorescence spectroscopy, which showed that the study of the small drug molecule was more practical and reasonable. The rationality of the experimental results obtained was verified by ultraviolet absorption spectroscopy.
\end{abstract}

Keywords: Cefetamet Pivoxil, Pepsin, Fluorescence Method, Improved Spectroscopy, Mechanism of Action

\section{Introduction}

In traditional fluorescence spectroscopy, the fluorescence of protein is mainly caused by tryptophan residues and the information of other non-fluorescent amino acid residues interacting with drugs in the protein cannot be reflected in the traditional fluorescence spectrum [1]. The spectrum can only reflect part of the information of the interaction between the entire protein molecule and the drug, resulting in inaccurate and one-sided information obtained. But the spectrum of small drug molecules can reflect the overall information, which changes can reflect the complete information of drug and protein. Because of this, a new method by taking the drug as the object of detection was applied to study the interaction between drugs and proteins and the results obtained were more accurate and reliable.

Pepsin (PEP), molecular weight of about 35,000, the first animal enzyme discovered by Theodor Schwann in 1836, is one of the aspartic acid protease and is a digestive protease
[2]. PEP is a proteolysis enzyme produced by the body's stomach and it can be used as a digestive drug, as well as for protein structure analysis. Cefetamet pivoxil (Cefetamet pivoxil, referred to as CFP) is the third generation cephalosporin, which antibacterial spectrum is wider than the first and second generation cephalosporin. It mainly used in clinical infection caused by a variety of sensitive bacteria, such as ENT, lower respiratory tract, urinary tract, skin and soft tissue [3]. In this paper, the mechanism of action of PEP-CFP system was studied by resonance light scattering method, and the rationality of the experimental results obtained was tested by ultraviolet absorption spectroscopy. The results showed that compared with the traditional protein, the drug as the test object could express the protein-drug interaction information comprehensively and accurately. The new method not only proposed to improve the mechanism of protein and drug binding but also helped to provide a theoretical basis for people to understand the mechanism of drug-protein interaction comprehensively and accurately. 


\section{Experimental}

\subsection{Apparatus and Materials}

All fluorescence spectra were recorded with a Shimadzu RF-5301PC. Absorption was measured with an UV-visible recording spectrophotometer (UV-3600 Shimadzu, Japan). All pH measurements were made with a pHS-3C precision acidity meter (Leici, Shanghai). All temperatures were controlled by a SYC-15B super-heated water bath (Nanjing Electronic equipment Factory)

A PEP (Sigma Company.) solution $\left(1.0 \times 10^{-4} \mathrm{~mol} \cdot \mathrm{L}^{-1}\right)$ was prepared. CFP standard solution (CAS\#, 64485-93-4) $\left(1.0 \times 10^{-3} \mathrm{~mol} \cdot \mathrm{L}^{-1}\right)$ was prepared Tris- $\mathrm{HCl}$ buffer solution containing $\mathrm{NaCl}\left(0.15 \mathrm{~mol} \cdot \mathrm{L}^{-1}\right)$ was used to keep the $\mathrm{pH}$ of the solution at 7.40. All other reagents were analytical grade and all aqueous solutions were prepared with newly double-distilled water and stored at $277 \mathrm{~K}$.

The fluorescence intensity measured in the experiment was corrected by the formula of internal filter effect [4].

$$
F_{c o r}=F_{o b s} \times e^{\left(A_{e x}+A_{e m}\right) / 2}
$$

where, $F_{c o r}$ and $F_{o b s}$ are the corrected and observed fluorescence intensities, respectively. $A_{\mathrm{ex}}$ and $A_{\mathrm{em}}$ are the absorbance values of the system at excitation and emission wavelengths, respectively.

\subsection{Procedures}

\subsubsection{Classical Fluorescence Spectroscopy Measurements}

$1.0 \mathrm{~mL}$ of $\mathrm{pH}=7.40$ Tris- $\mathrm{HCl}, 1.0 \mathrm{~mL}$ of PEP solution $\left(1.0 \times 10^{-5} \mathrm{~mol} \cdot \mathrm{L}^{-1}\right)$ and different volume of CFP were added into $10 \mathrm{~mL}$ colorimetric tube successively. The samples were diluted to scaled volume of $5 \mathrm{~mL}$ with water, mixed thoroughly by shaking, and kept static at different temperatures $(298,303$ and $310 \mathrm{~K})$. Place the prepared solution in a $1 \mathrm{~cm}$ quartz colorimetric dish. The excitation wavelength for the fluorescence spectra of PEP-CFP was 280 $\mathrm{nm}$ with the excitation and emission slit widths set at $5 \mathrm{~nm}$.

\subsubsection{Resonance Light Scattering Spectroscopy}

At 298, 303 and $310 \mathrm{~K}, 1.0 \mathrm{~mL}$ of Tris-HCl buffer, $\mathrm{pH}=7.40,1.0 \mathrm{~mL}$ of $1.0 \times 10^{-5} \mathrm{~mol} \cdot \mathrm{L}^{-1} \mathrm{CFP}$ solution and different volume of PEP solution were added into $10 \mathrm{~mL}$ colorimetric tube successively. The samples were diluted to scaled volume of $5 \mathrm{~mL}$ with double-distilled water. The fluorescence spectra were measured $(\Delta \lambda$ at $0 \mathrm{~nm}$ and emission wavelengths of 200-700 nm). The widths of both excitation and emission slit were set to $5 \mathrm{~nm}$.

\subsubsection{UV-Visible Measurements}

At 298,303 and $310 \mathrm{~K}, 1 \mathrm{~mL}$ of Tris- $\mathrm{HCl}$ buffer, $\mathrm{pH}=7.40$, $1.5 \mathrm{~mL}$ of $1.0 \times 10^{-4} \mathrm{~mol} \cdot \mathrm{L}^{-1} \mathrm{CFP}$ solution and different volume of PEP solution were added into $10 \mathrm{~mL}$ colorimetric tube successively, with corresponding concentration of PEP solution as the reference. The samples were diluted to scaled volume of $5.0 \mathrm{~mL}$ with double-distilled water and mixed thoroughly by shaking. The UV-visible absorption spectra of CFP in the presence and absence of PEP were scanned with 1 $\mathrm{cm}$ quartz cells in the range from 190 to $450 \mathrm{~nm}$ for 30 minutes and the absorption intensity $\mathrm{A}$ at the maximum absorption peak was recorded.

\section{Results and Discussion.}

\subsection{The Classical Fluorescence Spectra of PEP-CFP System}

The fluorescence spectra of PEP-CFP system was shown in Figure 1. As shown in Figure 1, the fluorescence intensity of PEP decreased gradually with the constant increase of CFP concentration (CFP non fluorescence). The result showed that there was interaction between CFP and PEP. The fluorescence quenching data were analyzed by Stern-Volmer eqn. [5] (2):

$$
I_{0} / I=1+K_{q} \tau_{0}[L]=1+K_{s v}[L]
$$

where, $I_{0}$ and $I$ are the fluorescence intensities of PEP in the absence and presence of the CFP, respectively. $K_{q}$ is the bimolecular quenching constant and $[L]$ is the concentration of the quencher, $\tau_{0}$ is the average lifetime of fluorescence and $K_{s v}$ is the Stern-Volmer quenching constant. The calculate results were shown in Table 1. From Table 1, it showed that the $K_{q}$ value was greater than $2.0 \times 10^{10} \mathrm{~L} \cdot \mathrm{mol}^{-1} \cdot \mathrm{s}^{-1}$ at different temperatures. The $K_{s v}$ values were inversely correlated with temperatures. The result showed that the combination process of PEP-CFP system was static quenching process [6].

Eqn. [7] (3) was used to calculate the binding constant $K_{a}$ and the number of binding sites $n$.

$$
\lg \left(\frac{I_{0}-I}{I}\right)=n \lg K_{a}+\mathrm{n} \lg \left\{\left[D_{t}\right]-n \frac{I_{0}-I}{I}\left[B_{t}\right]\right\}
$$

The binding parameters can be obtained by the plot of $1 \mathrm{~g}$ $\left(I_{0} / I-1\right)$ with $\lg \left\{\left[D_{\mathrm{t}}\right]-n\left[B_{\mathrm{t}}\right]\left(1-I / I_{0}\right)\right\}$. As shown in Table 1 , the fact that the values of $n$ were all approximately to 1 which implied that only one binding site between PEP and CFP. Meanwhile, the $K_{a}$ decreased with the rising temperature, further suggested that the quenching of the interaction between CFP and PEP was a static process [8].

Table 1. Quenching reactive parameters of PEP-CFP system at different temperatures.

\begin{tabular}{lllllll}
\hline$\lambda_{e x}(\mathbf{n m})$ & $\boldsymbol{T} /(\mathbf{K})$ & $\boldsymbol{K}_{q} /(\mathbf{L} / \mathbf{m o l} \cdot \mathbf{s})$ & $\boldsymbol{K}_{\boldsymbol{s}} /(\mathbf{L} / \mathbf{m o l})$ & $\boldsymbol{r}_{\boldsymbol{l}}$ & $\boldsymbol{K}_{\boldsymbol{a}} /(\mathbf{L} / \mathbf{m o l})$ & $\boldsymbol{n}$ \\
\hline \multirow{2}{*}{280} & 298 & $1.58 \times 10^{12}$ & $1.58 \times 10^{4}$ & 0.9983 & $1.46 \times 10^{4}$ & 1.12 \\
& 303 & $1.42 \times 10^{12}$ & $1.42 \times 10^{4}$ & 0.9989 & $1.35 \times 10^{4}$ & 1.09 \\
& 310 & $1.23 \times 10^{12}$ & $1.23 \times 10^{4}$ & 0.9963 & $1.17 \times 10^{4}$ & 1.08 \\
\hline
\end{tabular}

$r_{l}$ is the linear relative coefficient of $I_{0} / I \sim[L], r_{2}$ is the linear relative coefficient of $\lg \left[\left(I_{0}-I\right) / I\right] \sim \lg \left\{\left[D_{t}\right]-n\left[B_{t}\right]\left(I_{0}-I\right) / I_{0}\right\}$ 


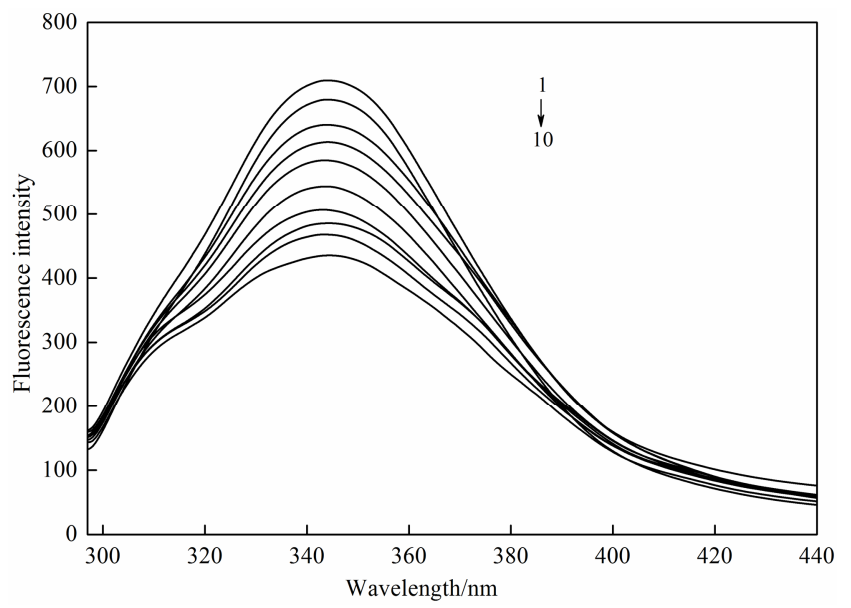

Figure 1. Fluorescence emission spectra of PEP-CFP $(T=298 \mathrm{~K})$, $C_{P E P}=2.0 \times 10^{-6} \mathrm{~mol} \cdot \mathrm{L}^{-1}, 1 \sim 10: C_{C F P}=(0,0.2,0.4,0.6,2.0,3.0,4.0,5.0,6.0,8.0)$ $\times 10^{-5} \mathrm{~mol} \cdot \mathrm{L}^{-1}$.

\subsection{Fluorescence Quenching Spectra of PEP-CFP System}

The fluorescence spectra of PEP-CFP system was shown in Figure 2. As shown in Figure 2, the fluorescence intensity of CFP decreased gradually with the addition of PEP with blue shift of $380 \mathrm{~nm}$. The result showed that PEP could quench the intrinsic fluorescence of CFP significantly and there was an interaction between PEP and CFP. It could also indicate that both tryptophan and tyrosine entered into the reaction indirectly [9].

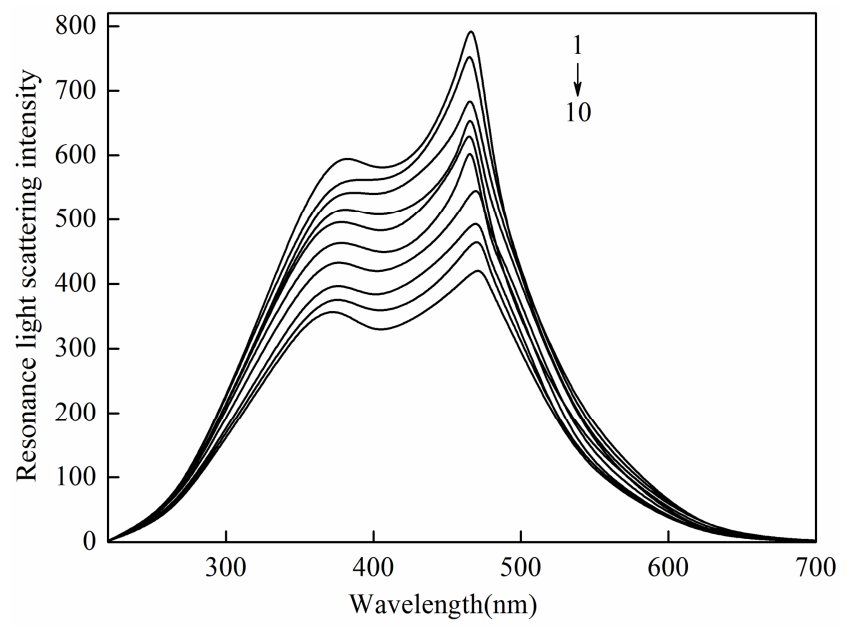

Figure 2. RLS spectra of PEP-CFP system $(T=298 \mathrm{~K}), C_{C F P}=2.0 \times 10^{-6}$ $\mathrm{mol} \cdot \mathrm{L}^{-1} ; 1 \sim 9: C_{P E P}=(0,0.2,0.4,0.6,1.0,2.0,3.0,4.0,6.0,8.0) \times 10^{-7} \mathrm{~mol} \cdot \mathrm{L}^{-1}$.

Table 2. RLS quenching reactive parameters of PEP-CFP system at different temperatures.

\begin{tabular}{llllll}
\hline $\boldsymbol{T} /(\mathbf{K})$ & $\boldsymbol{K}_{\boldsymbol{q} \mathbf{l}} /(\mathbf{L} / \mathbf{m o l} \cdot \mathbf{s})$ & $\boldsymbol{K}_{\boldsymbol{s v} \mathbf{l}} /(\mathbf{L} / \mathbf{m o l})$ & $\boldsymbol{r}_{\boldsymbol{3}}$ & $\boldsymbol{K}_{\boldsymbol{a} 1} /(\mathbf{L} / \mathbf{m o l})$ & $\boldsymbol{n}$ \\
\hline 298 & $2.95 \times 10^{14}$ & $2.95 \times 10^{6}$ & 0.9991 & $3.25 \times 10^{6}$ & 1.08 \\
303 & $1.77 \times 10^{14}$ & $1.77 \times 10^{6}$ & 0.9972 & $2.79 \times 10^{6}$ & 0.9985 \\
310 & $1.02 \times 10^{14}$ & $1.02 \times 10^{6}$ & 0.9988 & $2.24 \times 10^{6}$ & 0.9989 \\
\hline
\end{tabular}

$r_{3}$ is the linear relative coefficient of $I_{0} / I \sim[L] ; \mathrm{r}_{4}$ is the linear relative coefficient of $\lg \left[\left(I_{0}-I\right) / I\right] \sim \lg \left\{\left[D_{t}\right]-n\left[B_{t}\right]\left(I_{0}-I\right) / I_{0}\right\}$

Be calculated by equation eqn. (2) (3), the calculated result was shown in Table 2. As shown in Table 2, the fact that the values of $n$ were all approximately to 1 , which implied that just one binding site for CFP existed in PEP. Meanwhile, the $K_{a l}$ and $K_{s v l}$ were both decreased with the rising temperature, further suggested that the quenching was a static process, which were consistent with the results of classical fluorescence spectroscopy. The quenching mechanism and quenching parameters obtained by the resonance scattering fluorescence spectroscopy method and the fluorescence quenching method were in agreement with each other, which indicated that the resonance scattering fluorescence spectroscopy method is feasible in studying the binding of drugs to proteins. The binding constant of the resonance scattering fluorescence spectroscopy method was two orders of magnitude larger than that of the fluorescence quenching method, which indicated that not only the tryptophan residues were involved in the peptide chain of PEP but also the other residues interact with CFP [10]. In addition to the "point to point" interaction between CFP and PEP, the "point to side" interaction between CFP and the other peptide in PEP hydrophobic region also existed [11]. Compared to classical fluorescence spectroscopy with protein as detection object, it was treating drugs as detection object could give more complete and accurate expression of the interaction information of protein and drugs.

\subsection{UV-Visible Spectra Studies}

The UV-visible absorption spectra of PEP-CFP were shown in Figure 3. The binding constant $K_{b}$ of protein and drug could be calculated on the following eqn. 4 [12-13]:

$$
\left(A_{0}-A\right)^{-1}=A_{0}^{-1}+K_{b}^{-1} A_{0}^{-1}[D]^{-1}
$$

where, $A_{0}$ and $A$ are the absorption values in the absence and presence of quencher, respectively. As shown in Figure 3, with gradual addition of PEP to CFP solution, the intensity of the peak at $199 \mathrm{~nm}$ decreased with a slight red shift, indicating that the interaction between PEP and CFP led to the formation of a complex between drug and protein and generated a new substance. Based on the linear regression plot of $\left(A_{0}-A\right)^{-1}$ versus $[L]^{-1}$, the $K_{b}$ values could be obtained. The calculated results were shown in Table 3. As seen in Table 3, the binding constant $K_{b}$ decreased with rising temperatures, which was consistent with the results of fluorescence methods. The $K_{b}$ values were observed to be much larger than $K_{a}$ of the classical fluorescence spectroscopy and closed to $K_{a l}$ obtained by resonance scattering fluorescence spectroscopy. This phenomenon also showed that treating the drug as detection objects could give more complete and accurate expression the interaction information of proteins and drugs. The difference between $K_{b}$ and $K_{a l}$ might be due to the difference between the two other research methods. 
Table 3. The binding constants of PEP-CFP system by UV absorption spectrometry at different temperatures.

\begin{tabular}{llll}
\hline $\boldsymbol{T} /(\mathbf{K})$ & $\boldsymbol{K}_{\boldsymbol{b}} / \mathbf{( \mathbf { L } / \mathbf { m o l } )}$ & Linear regression equation & $\boldsymbol{r}_{5}$ \\
\hline 298 & $1.86 \times 10^{6}$ & $\left(A_{0}-A\right)^{-1}=6.728+3.617 \times 10^{-6}[L]^{-1}$ & 0.9963 \\
303 & $1.61 \times 10^{6}$ & $\left(A_{0}-A\right)^{-1}=6.148+3.819 \times 10^{-6}[L]^{-1}$ & 0.9985 \\
310 & $1.34 \times 10^{6}$ & $\left(A_{0}-A\right)^{-1}=6.528+4.872 \times 10^{-6}[L]^{-1}$ & 0.9996 \\
\hline
\end{tabular}

$r_{5}$ is the linear relative coefficient of $\left(A_{0}-A\right)^{-1} \sim[L]^{-1}$

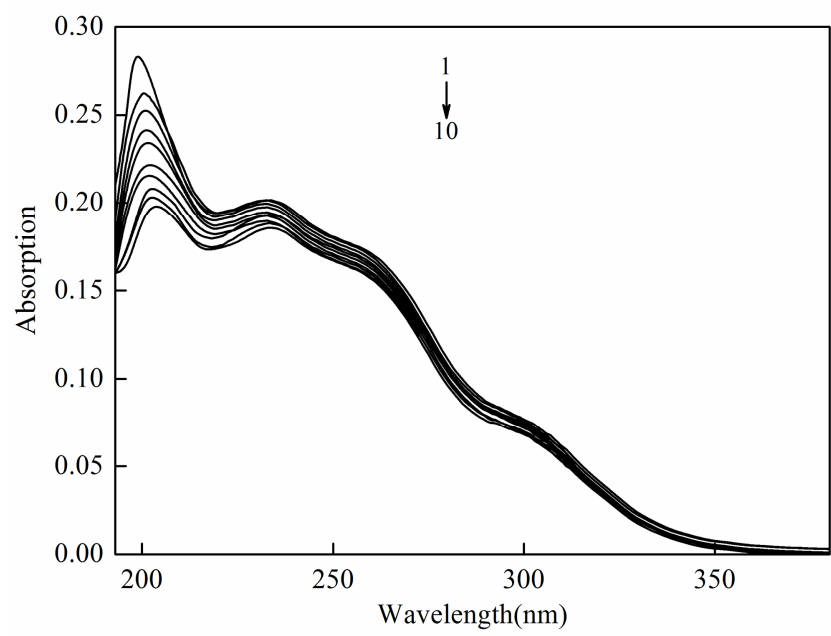

Figure 3. Absorption spectra of PEP-CFP system $(T=298 \mathrm{~K}), C_{C F P}=$ $3.0 \times 10^{-5} \mathrm{~mol} \cdot \mathrm{L}^{-1} ; 1 \sim 10: C_{P E P}=(0,1.0,2.0,3.0,4.0,5.0,6.0,7.0,8.0,9.0)$ $\times 10^{-7} \mathrm{~mol} \cdot \mathrm{L}^{-1}$.

\subsection{Type of Interaction Force of PEP-CFP System}

Generally, the interaction force between the small drug molecule and biological macromolecule includes hydrogen bond, Vander Waals force, electrostatic interactions and hydrophobic force, etc. The type of interaction force in PEP-CFP system can be obtained by the thermodynamic parameters of PEP-CFP, and calculated by formula (5) (6) [14]. The calculated results were shown in Table 4.

$$
\begin{gathered}
R \ln K=\Delta S-\Delta H / T \\
\Delta G=-R T \ln K=\Delta H-T \Delta S
\end{gathered}
$$

The values of thermodynamic parameters $\mathrm{K}, \Delta H, \Delta S$ and $\Delta G$ were listed in Table 4 . Many researchers thought that a negative value for $\Delta H$ and a positive value for $\Delta S$ indicated that electrostatic force had a major role in the binding reaction. Besides, the results from the negative value of $\Delta G$ clarified that there had been a spontaneous reaction between PEP and CFP [15]. A negative $\Delta H$ and positive $\Delta S$ showed that the electrostatic interaction played a major role in the binding process [16]. The same conclusion of the three experimental methods indicated that when researched the type of interaction between drugs and proteins, the resonance light scattering method, ultraviolet absorption method and the traditional fluorescence quenching method were both feasible. It was more obvious that the thermodynamic parameters obtained by the resonance light scattering method and the ultraviolet absorption method were close to each other, indicating that fixed drug concentration to study the mechanism of drug-protein interaction was a priority experimental scheme.

\begin{tabular}{|c|c|c|c|c|c|}
\hline & $T /(\mathbf{K})$ & $K_{a} /\left(\mathrm{L} \cdot \mathrm{mol}^{-1}\right)$ & $\Delta H /\left(\mathrm{KJ} \cdot \mathrm{mol}^{-1}\right)$ & $\Delta S /\left(J \cdot \mathrm{mol}^{-1} \cdot \mathrm{K}^{-1}\right)$ & $\Delta G /\left(\mathrm{KJ} \cdot \mathrm{mol}^{-1}\right)$ \\
\hline \multirow{3}{*}{$\begin{array}{l}\text { Classical fluorescence } \\
\text { spectroscopy }\end{array}$} & 298 & $1.46 \times 10^{4}$ & \multirow{3}{*}{-14.27} & 31.83 & -23.76 \\
\hline & 303 & $1.35 \times 10^{4}$ & & 31.97 & -23.96 \\
\hline & 310 & $1.17 \times 10^{4}$ & & 31.84 & -24.14 \\
\hline \multirow{3}{*}{$\begin{array}{l}\text { Elastic scattering fluorescence } \\
\text { spectroscopy }\end{array}$} & 298 & $3.25 \times 10^{6}$ & \multirow{3}{*}{-23.86} & 44.60 & -37.15 \\
\hline & 303 & $2.79 \times 10^{6}$ & & 44.65 & -37.39 \\
\hline & 310 & $2.24 \times 10^{6}$ & & 44.61 & -37.69 \\
\hline \multirow{3}{*}{ UV-vis absorption spectroscopy } & 298 & $1.86 \times 10^{6}$ & \multirow{3}{*}{-20.96} & 49.69 & -35.77 \\
\hline & 303 & $1.61 \times 10^{6}$ & & 49.65 & -36.00 \\
\hline & 310 & $1.34 \times 10^{6}$ & & 49.69 & -36.36 \\
\hline
\end{tabular}

Table 4. The thermodynamic parameters of PEP-CFP system at different temperatures.

\subsection{Drug Cooperativity}

The binding of the receptor PEP to the ligand CFP was analyzed by using Hill coefficient $n_{H}$ to compare with 1 . According to formula (7) and (8) [17], it could obtain the $n_{H}$ value of PEP-CFP system.

$$
\lg \frac{Y}{1-Y}=\lg K+n_{H} \lg [D]
$$

where, $K$ is the binding constant; $Y$ is the fractional binding saturation; $n_{H}$ is the Hill's coefficient.

$$
\frac{Y}{1-Y}=\frac{Q}{Q_{m}-Q}
$$

In the formula (8), $Q$ is $\left(F_{0}-F\right) / F_{0} . Q_{m}$ is intercept of the plot $1 / Q$ versus $1 /[D]$. Hill's coefficient $n_{\mathrm{H}}$ of PEP-CFP system can be gained from the slope of the plot of $\lg [Y /$ $(1-Y)]$ versus $\lg [D]$. The results were presented in Table 5. From Table 5, it could be seen that the values of $n_{H}$ were equal to 1 approximately at three different temperatures by the three methods, which indicated that there was no cooperative reaction between PEP and CFP [18]. It further suggested that resonance scattering fluorescence 
spectroscopy and UV-visible spectroscopy was correct to determine cooperative between drug and protein.

Table 5. Hill coefficient of PEP-CFP system at different temperatures.

\begin{tabular}{lllllll}
\hline \multirow{2}{*}{$/(\mathbf{K})$} & \multicolumn{2}{l}{ Classical fluorescence spectroscopy } & \multicolumn{2}{c}{ Elastic scattering fluorescence spectroscopy } & \multicolumn{2}{l}{ UV-vis absorption spectroscopy } \\
\cline { 2 - 7 } & $\boldsymbol{n}_{\boldsymbol{H}}$ & $\boldsymbol{r}_{\boldsymbol{6}}$ & $\boldsymbol{n}_{\boldsymbol{H}}$ & $\boldsymbol{n}_{\boldsymbol{6}}$ & $\boldsymbol{n}_{\boldsymbol{H}}$ & 0.996 \\
\hline 298 & 1.054 & 0.9946 & 1.082 & 0.9994 & 0.996 & 0.9965 \\
310 & 1.041 & 0.9968 & 0.994 & 0.9965 & 1.042 & 0.9991 \\
318 & 1.045 & 0.9983 & 0.997 & 0.9979 & 0.990 & 0.9947 \\
\hline
\end{tabular}

$r_{6}$ is the linear relative coefficient of $\lg [Y /(1-Y)] \sim \lg [D]$

\section{Conclusion}

The interaction between PEP and CFP was studied by fluorescence spectroscopy, resonance fluorescence scattering and ultraviolet absorption spectroscopy. Compared the binding constants of the three methods, there were obvious differences in calculating the binding constants of the system. The $K_{a}$ values of classical fluorescence spectroscopy were smaller than resonance fluorescence scattering method and ultraviolet absorption method. Although there were some differences between the resonance fluorescence scattering method and the ultraviolet absorption method, the difference was minimal. Therefore, it was more comprehensive and accurate to present the interaction between drugs and proteins. Improved spectroscopy was an innovation based on the traditional fluorescence method, which provided a new way to study the interaction more accurately between drugs and proteins. It would further improve the study of the reaction mechanism between drugs and proteins.

\section{Acknowledgements}

The authors gratefully acknowledge the financial support of National Natural Science Foundation of China (No. 21375032).

\section{References}

[1] T Tan, R Huang, and Z H Xia, Chinese J Anal Chem, 2007, 35 , $1415-1420$.

[2] O C Aszmann, J Reconstr Microsurg, 2000, 16, 291-295.
[3] K Noh, E Kim, and W Kang, Biomed Chromatogr, 2011, 25, 779-782.

[4] R F Steiner, and I Weinryb, Springer US, 1971.

[5] J Wang, C Chan, F W Huang, et al. Med Chem Res, 2016, 26, $1-9$.

[6] R Huang, S Zhang, L Pan, et al. Spectrochim Acta Part A, 2013, 104, 377-382.

[7] Y Sun, S Wei, C Yin, et al. J Bioorg Med Chem Lett, 2011, 21, 3798-3804 (2011).

[8] S Ghosh, S Jana, and N Guchhait, J Phys Chem B, 2012, 116, 1155-1163.

[9] AS Sharma, S Anandakumar, and M Ilanchelian, J Lumin, 2014, 151, 206-218.

[10] C Jash, P V Payghan, N Ghoshal, et al. J Phys Chem B, 2014, 118, No. 46, 13077-13091 (2014).

[11] S Bi, Y Sun, C Qiao, et al. J Lumin, 2009, 129, 541-547.

[12] M He, Z Xia, Y Yin, et al. Chin. J. Mod. Appl. Pharm, 2004, 21, 429-432.

[13] R Han, B Liu, G Li, et al. J Lumin, 2016, 31, 580-586.

[14] W Wang, W Min, J Chen, et al. J Lumin, 2011, 131, 820-824.

[15] B Kaboudin, K Moradi, M R Faghihi, et al. J Lumin, 2013, 139, 104-112.

[16] X Hu, Z Deng, Y Wang, et al. Soft Mater, 2014, 12, 277-283.

[17] B Bojko, A Sułkowska, M Maciążek-Jurczyk, et al. Spectrochim Acta A, 2010, 52, 384-390.

[18] L Shen, H Xu, F Huang, et al. Spectrochim Acta A, 2015, 135, 256-263. 